\title{
ISOMORPHISM VERSUS COMMENSURABILITY FOR A CLASS OF FINITELY PRESENTED GROUPS
}

\author{
GOULNARA ARZHANTSEVA, JEAN-FRANÇOIS LAFONT, AND ASHOT MINASYAN
}

\begin{abstract}
We construct a class of finitely presented groups where the isomorphism problem is solvable but the commensurability problem is unsolvable. Conversely, we construct a class of finitely presented groups within which the commensurability problem is solvable but the isomorphism problem is unsolvable. These are first examples of such a contrastive complexity behavior with respect to the isomorphism problem.
\end{abstract}

\section{INTRODUCTION}

The purpose of this paper is to study the relative algorithm complexities of the following two major group theoretical decision problems: the isomorphism problem and the commensurability problem.

Both of these problems have a long history [12, 26], a meaningful topological interpretation [27, 5], and a number of famous solutions for specific classes of groups [13, 20, 21]. However, their comparison from the algorithmic point of view seems not to have been done up to now. Moreover, there have been numerous results comparing decision problems dealing with elements in a single group, such as the word problem, conjugacy problem, power problem, etc. (see, for instance, [18, 21]). In contrast, there have so far been no comparative results involving the isomorphism problem. We remedy this situation, by establishing the following two complementary theorems:

Theorem 1.1. There exists a recursively enumerable class $\mathscr{C}_{1}$ of finite presentations of groups, with uniformly solvable word problem, such that the isomorphism problem is solvable but the commensurability problem is unsolvable within this class.

Theorem 1.2. There exists a recursively enumerable class $\mathscr{C}_{2}$ of finite presentations of groups such that the commensurability problem is solvable but the isomorphism problem is unsolvable within this class.

These results are all the more unexpected as Thomas [30, Thm. 1.1] showed that the isomorphism and commensurability problems have the same complexity from the viewpoint of descriptive set theory.

Let us now explain the terminology and the meaning of our main theorems.

Date: September 19, 2011.

2000 Mathematics Subject Classification. Primary 20F10; Secondary 20F69, 03D40, 20 F65.

Key words and phrases. Commensurable groups, virtual isomorphism problem, groups without finite quotients. 
A class $\mathscr{C}$ of finite presentations of groups has uniformly solvable word problem if there is an algorithm which takes as input a presentation $P \in \mathscr{C}$ and a word in the generators of this presentation, and decides whether or not this word represents the identity element of the group given by $P$.

Two groups $G_{1}, G_{2}$ are commensurable if there exist two subgroups of finite index $H_{i} \leqslant G_{i}$ for $i=1,2$, such that $H_{1}$ and $H_{2}$ are isomorphic. It is not difficult to see that commensurability is an equivalence relation.

Given a class $\mathscr{C}$ of finite presentations of groups, we say that the isomorphism problem is solvable within $\mathscr{C}$ [commensurability problem is solvable within $\mathscr{C}$ ] if there is an algorithm, taking on input two group presentations from $\mathscr{C}$ and deciding whether or not these presentations define isomorphic [commensurable] groups.

Often, when considering the isomorphism problem, one is looking at a certain class $\mathscr{G}$ of finitely presented groups. This actually means the class of all finite presentations of groups from $\mathscr{G}$. At first glance, it might seem that our Theorems 1.1 and 1.2 are somewhat more restrictive, as we are only picking out some specific family of presentations. Let us clarify this issue.

Let $\mathscr{G}_{1}$ denote the collection of all groups defined via the presentations in the class $\mathscr{C}_{1}$ appearing in Theorem 1.1 and let $\hat{\mathscr{C}}_{1}$ denote the class of all finite presentations of groups from $\mathscr{G}_{1}$ (so clearly $\mathscr{C}_{1} \subset \widehat{\mathscr{C}}_{1}$ ). It follows immediately from Theorem 1.1 that the commensurability problem is unsolvable for the class $\widehat{\mathscr{C}}_{1}$ of finite presentations of groups, as it is already unsolvable within the subclass $\mathscr{C}_{1}$. On the other hand, the isomorphism problem is still solvable within the class $\widehat{\mathscr{C}}_{1}$. Indeed, given any presentation $P \in \widehat{\mathscr{C}}_{1}$, one can start applying Tietze transformations to it; simultaneously we can start writing down the finite presentations from $\mathscr{C}_{1}$, because the class $\mathscr{C}_{1}$ is recursively enumerable. At each step we can compare the transformations of $P$, obtained so far, with the presentations from the class $\mathscr{C}_{1}$, written down by this step. After finitely many steps we will find a finite presentation $P^{\prime} \in \mathscr{C}_{1}$ which defines the same group (up to isomorphism) as $P$ (see [19, II.2.1])). This easily yields an algorithm that identifies a pair of presentations from $\mathscr{C}_{1}$ which define the same groups as the given pair of presentations in $\widehat{\mathscr{C}}_{1}$. Taking the resulting pair of presentations in $\mathscr{C}_{1}$, we can then apply the algorithm for deciding the isomorphism problem within the subclass $\mathscr{C}_{1}$. As such, we view Theorem 1.1 as a statement about the corresponding class of groups $\mathscr{G}_{1}$.

Similarly, let $\mathscr{G}_{2}$ denote the collection of all groups defined via the presentations in the class $\mathscr{C}_{2}$ appearing in Theorem 1.2 and let $\widehat{\mathscr{C}}_{2}$ denote the class of all finite presentations of groups from $\mathscr{G}_{2}$ (so again, we have $\mathscr{C}_{2} \subset \widehat{\mathscr{C}}_{2}$ ). By an argument, identical to the one in the previous paragraph, we have that the isomorphism problem is unsolvable in the class $\widehat{\mathscr{C}}_{2}$, but the commensurability problem is solvable. This allows us to view Theorem 1.2 as a statement about the corresponding class of groups $\mathscr{G}_{2}$.

The fact that the isomorphism problem is unsolvable within the class $\mathscr{C}_{2}$ implies, in particular, that there are infinitely many pairwise non-isomorphic groups within $\mathscr{G}_{2}$. More precisely, the set of representatives of isomorphism classes of groups from $\mathscr{G}_{2}$ is 
not recursively enumerable. This fact is of particular interest because it cannot be seen directly from our construction of the class $\mathscr{C}_{2}$ below.

The proofs of both theorems rely on a combination of various embedding theorems from combinatorial and geometric group theory involving finitely presented infinite simple groups and infinite groups with no finite quotients. The main idea is to start with a single group $G$ and construct a class $\mathscr{K}$, of mapping tori of $G$, for which the isomorphism problem is directly related to the word problem in $G$. Similarly, the commensurability problem in $\mathscr{K}$ will be directly related to the torsion problem in $G$. Thus the solvability/unsolvability of the word [resp. torsion] problem in $G$ will yield the same for the isomorphism [resp. commensurability] problem in $\mathscr{K}$.

Besides isomorphism and commensurability, there are other natural equivalence relations on the class of finitely presented groups such as virtual isomorphism, bi-Lipschitz equivalence, quasi-isometry, etc. We discuss the corresponding algorithmic problems in the last section, where we also state some open questions.

\section{MAPPING TORI OF GROUPS WITHOUT PROPER FINITE INDEX SUBGROUPS}

Let $G$ be a group and $\varphi \in \operatorname{Aut}(G)$ be an automorphism of $G$. Let $G_{\varphi}:=G \rtimes_{\varphi} \mathbb{Z}$ denote the associated mapping torus. As a set, $G_{\varphi}=G \times \mathbb{Z}$ and the group product is defined by $(g, n)\left(g^{\prime}, m\right):=\left(g \cdot \varphi^{n}\left(g^{\prime}\right), n+m\right)$, where $\varphi^{n}$ denotes the automorphism of $G$ which is the $n$-fold composition of $\varphi$, with the convention that $\varphi^{0}=i d_{G}$, where $i d_{G} \in \operatorname{Aut}(G)$ the identity automorphism of $G$.

We shall consider the class of groups $\mathscr{K}_{G, \Phi}=\left\{G_{\varphi} \mid \varphi \in \Phi\right\}$, where $\Phi$ is some subset of $\operatorname{Aut}(G)$, and analyze the isomorphism problem within the corresponding class of group presentations. We denote by $\bar{\varphi}$ the image of $\varphi$ under the canonical epimorphism $\operatorname{Aut}(G) \rightarrow \operatorname{Out}(G):=\operatorname{Aut}(G) / \operatorname{Inn}(G)$ onto the quotient of $\operatorname{Aut}(G)$ by the subgroup consisting of inner automorphisms.

Proposition 2.1. Suppose that $G$ is a group which has no epimorphisms onto $\mathbb{Z}$, and $\varphi, \psi \in \operatorname{Aut}(G)$. Then the following are equivalent.

(i) $G_{\varphi}$ is isomorphic to $G_{\psi}$;

(ii) $\bar{\varphi} \in \operatorname{Out}(G)$ is conjugate to one of the two elements $\bar{\psi}, \bar{\psi}^{-1} \in \operatorname{Out}(G)$.

Proof. Suppose that $G_{\varphi}$ is isomorphic to $G_{\psi}$ via an isomorphism

$$
\rho: G \rtimes_{\varphi} \mathbb{Z} \longrightarrow G \rtimes_{\psi} \mathbb{Z} \text {. }
$$

Let $\tau: G \rightarrow \mathbb{Z}$ be the homomorphism defined by the composition

$$
G \hookrightarrow G \rtimes_{\varphi} \mathbb{Z} \stackrel{\rho}{\longrightarrow} G \rtimes_{\psi} \mathbb{Z} \rightarrow \mathbb{Z},
$$

with the natural inclusion and epimorphism maps. It follows that $\tau$ is trivial as by hypothesis we know that $G$ does not map onto $\mathbb{Z}$. Therefore, the restriction of $\rho$ to $G$ has image entirely contained in $G \leqslant G_{\psi}$. Applying the same argument to $\rho^{-1}$ and recalling that $\rho^{-1} \circ \rho=i d_{G_{\varphi}}$, we can conclude that $\rho$ maps the $G$-factor in $G_{\varphi}$ isomorphically onto the $G$-factor in $G_{\psi}$. 
On the other hand, a generator $t$ of the $\mathbb{Z}$-factor in $G_{\varphi}=G \rtimes_{\varphi} \mathbb{Z}$ has to map to a generator under the composition

$$
\langle t\rangle=\mathbb{Z} \hookrightarrow G \rtimes_{\varphi} \mathbb{Z} \stackrel{\rho}{\longrightarrow} G \rtimes_{\psi} \mathbb{Z} \rightarrow \mathbb{Z} .
$$

Indeed, the composition map $G_{\varphi} \stackrel{\rho}{\longrightarrow} G_{\psi} \rightarrow \mathbb{Z}$ is surjective as $\rho$ is an isomorphism. Since $G \leqslant G_{\varphi}$ is contained in the kernel of this map, the image is determined by the image of the quotient group $G_{\varphi} / G$. However, such an image coincides with $\langle t\rangle$ through the short exact sequence $\{1\} \rightarrow G \hookrightarrow G_{\varphi} \rightarrow \mathbb{Z} \rightarrow\{1\}$. Thus, the surjectivity of $G_{\varphi} \stackrel{\rho}{\longrightarrow}$ $G_{\psi} \rightarrow \mathbb{Z}$ implies that $t \in G_{\varphi}$ maps to a generator $s^{ \pm 1}$ of the $\mathbb{Z}$-factor in $G_{\psi}$.

Thus, in terms of splittings, the isomorphism $\rho$ is of the form:

$$
\begin{array}{lll}
(x, 0) & \stackrel{\rho}{\mapsto} & (\alpha(x), 0) \\
(e, 1) & \stackrel{\rho}{\mapsto} & (g, \pm 1)
\end{array}
$$

for any $x \in G$ and some fixed $\alpha \in \operatorname{Aut}(G)$ and $g \in G$ (e $e \in G$ is the identity element).

Let us now focus on the case where $(e, 1) \stackrel{\rho}{\longmapsto}(g, 1)$. Since the map $\rho$ is assumed to be an isomorphism, it must preserve the relations of the group $G_{\varphi}$. Evaluating $\rho$ on the relation $(e, 1)(x, 0)(e, 1)^{-1}=(\varphi(x), 0)$ yields the required constraint on the automorphisms. Indeed, evaluating the left hand side, we obtain

$$
\rho\left((e, 1)(x, 0)(e, 1)^{-1}\right)=(g, 1)(\alpha(x), 0)\left(\psi^{-1}\left(g^{-1}\right),-1\right)=\left(g \psi(\alpha(x)) g^{-1}, 0\right),
$$

while evaluating the right hand side, we obtain

$$
\rho((\varphi(x), 0))=(\alpha(\varphi(x)), 0)) .
$$

We deduce that the automorphism $\alpha \in \operatorname{Aut}(G)$ and the element $g \in G$ are related to the given automorphisms $\varphi, \psi \in \operatorname{Aut}(G)$ as follows:

$$
\alpha \circ \varphi=c_{g} \circ \psi \circ \alpha,
$$

where $c_{g} \in \operatorname{Aut}(G)$ is the inner automorphism defined by $c_{g}(y)=g y g^{-1}$ for all $y \in G$.

Passing to the outer automorphism group, we see that we have to have $\bar{\alpha} \circ \bar{\varphi}=\bar{\psi} \circ \bar{\alpha}$, that is, the classes $\bar{\varphi}$ and $\bar{\psi}$ are conjugate in $\operatorname{Out}(G)$.

Conversely, if the classes $\bar{\varphi}, \bar{\psi} \in \operatorname{Out}(G)$ are conjugate by some $\bar{\alpha} \in \operatorname{Out}(G)$, then one can find an element $g \in G$ so that $\alpha \circ \varphi=c_{g} \circ \psi \circ \alpha$. It is now immediate that $G_{\varphi} \cong G_{\psi}$, via the isomorphism map defined by $(x, 0) \mapsto(\alpha(x), 0)$ and $(e, 1) \mapsto(g, 1)$.

A similar analysis can be done in the case $(e, 1) \stackrel{\rho}{\longmapsto}(g,-1)$. This yields the relation $\alpha \circ \varphi=c_{g} \circ \psi^{-1} \circ \alpha$, that is, the classes $\bar{\varphi}$ and $\bar{\psi}^{-1}$ are conjugate in $\operatorname{Out}(G)$. This finishes the proof.

In order to facilitate the notation let us give the following

Definition 2.2. We will say that group $G$ is NFQ ('No Finite Quotients'), if the only finite quotient of $G$ is the trivial group. 
Since every finite index subgroup contains a finite index normal subgroup, a group $G$ is NFQ if and only if $G$ has no proper subgroups of finite index. It is easy to see that any NFQ group $G$ has no epimorphisms onto $\mathbb{Z}$, and thus it satisfies the assumptions of Proposition 2.1. Basic examples of NFQ groups are infinite simple groups.

To study the commensurability problem within the class $\mathscr{K}_{G, \Phi}$, we need to know the structure of subgroups of finite index in the corresponding mapping tori. The following observation shows that all such subgroups are "congruence subgroups":

Proposition 2.3. Let $G$ be a NFQ group, and $\varphi \in \operatorname{Aut}(G)$. Let $\pi: G_{\varphi} \rightarrow \mathbb{Z}$ be the canonical projection onto the $\mathbb{Z}$-factor of the mapping torus. Assume that $H \leqslant G_{\varphi}$ is a finite index subgroup of $G_{\varphi}$. Then $H=\pi^{-1}(k \mathbb{Z}) \cong G_{\varphi^{k}}$, where $k$ is the index of $H$ in $G_{\varphi}$ (and in particular, $H$ must be normal in $G_{\varphi}$ ).

Proof. By the assumptions, $\left[G_{\varphi}: H\right]<\infty$, hence $[G:(G \cap H)]<\infty$, therefore ker $\pi=$ $G \leqslant H$ as $G$ is NFQ. This forces $H$ to be of the form $\pi^{-1}(k \mathbb{Z})$ for some $k$. The value of $k$ can then be easily deduced:

$$
k=[\mathbb{Z}: k \mathbb{Z}]=\left[G_{\varphi}: \pi^{-1}(k \mathbb{Z})\right]=\left[G_{\varphi}: H\right],
$$

as stated in the proposition.

Combining Propositions 2.1 and 2.3, we immediately obtain

Corollary 2.4. Let $G$ be a NFQ group, and $\varphi \in \operatorname{Aut}(G)$. Then $G_{\varphi}$ is commensurable with $G_{i d_{G}} \cong G \times \mathbb{Z}$ if and only if the element $\bar{\varphi} \in \operatorname{Out}(G)$ has finite order.

Given two groups $A$ and $B$, consider their free product $G=A * B$. For any element $a \in A$ we can define a natural automorphism $\tau_{a} \in \operatorname{Aut}(G)$ by $\tau_{a}(x):=a^{-1} x a$ for all $x \in A$ and $\tau_{a}(y):=y$ for all $y \in B$. Note that $\left(\tau_{a}\right)^{k}=\tau_{a^{k}}$ in $\operatorname{Aut}(G)$ for all $k \in \mathbb{Z}$.

Lemma 2.5. Suppose that $B \neq\{1\}, a \in A$ and $G=A * B$. Then $\tau_{a} \in \operatorname{Inn}(G)$ if and only if a belongs to the center of $A$.

Proof. Clearly, if $a$ is central in $A$, then $\tau_{a}=i d_{G} \in \operatorname{Inn}(G)$. Conversely, suppose that there is $c \in A$ such that $a^{-1} c a \neq c$ in $A$. Take any $b \in B \backslash\{1\}$ and consider the element $g:=c b \in G$. Then $\tau_{a}(g)=a^{-1} c a b$ is not conjugate to $g$ in $G=A * B$ by the criterion of conjugacy in free products (see [19, IV.1.4]). Hence $\tau_{a} \notin \operatorname{Inn}(G)$, as required.

Since the free product of two NFQ groups is again a NFQ group, we can put together Proposition 2.1. Corollary 2.4 and Lemma 2.5 to achieve

Corollary 2.6. Let $A$ and $B$ be NFQ groups such that $B \neq\{1\}$ and $A$ has trivial center. Then for $G=A * B$ and any $a \in A$ the following are true:

- $G_{\tau_{a}}$ is isomorphic to $G_{i d_{G}}$ if and only if $a=1$ in $A$;

- $G_{\tau_{a}}$ is commensurable with $G_{i d_{G}}$ if and only if a has finite order in A. 


\section{WORD AND TORSION PROBLEMS IN NFQ GROUPS}

For a finite set $X$, we use $X^{*}$ to denote the set of words with letters from $X^{ \pm 1}$. Let $R$ be a set of words from $X^{*}$ and suppose that $G$ is a group given by the presentation $P=\langle X \| R\rangle$.

For a subset $Z \subseteq X^{*}$, we say the word problem for $Z$ in $G$ is solvable if there is an algorithm, which takes on input a word $w \in Z$ and decides whether or not this word represents the identity element of $G$. If $Z=X^{*}$, then the word problem for $Z$ in $G$ is simply known as the word problem in $G$. The word problem is one of the three fundamental group-theoretical decision problems introduced by Max Dehn [12] in 1911 (other two being the conjugacy and the isomorphism problems). It is well known that if the word problem for $G$ is solvable with respect to one finite generating set, then it is solvable with respect to any other finite generating set of $G$.

For an arbitrary subset $Z \subseteq X^{*}$, one can also consider the torsion problem for $Z$ in $G$, asking whether there exists an algorithm which inputs a word $w \in Z$, and decides whether or not $w$ represents an element of finite order in $G$. This is closely related to some decision problems considered by Lipschutz and Miller in [18] (for instance, it is a special case of the power problem).

Proposition 3.1. Every finitely presented group $H$ can be embedded into a finitely presented NFQ group A with trivial center. Moreover, if the word problem in $H$ is solvable then it is also solvable in $A$.

Proof. Take any infinite finitely presented simple group $S$ (for instance, Thompson's group $T$ or $V$ [17], or see [6, 7, 8, 9] for other such groups) and consider the free product $G=S * S$. Then $G$ is NFQ and hyperbolic relative to these two copies of $S$. Therefore, by Theorem 1.1 from [3], $H$ can be isomorphically embedded into some quotient $Q$ of $G$. Moreover, from the proof of this theorem, it follows that $Q$ can be obtained from $H * G$ by adding only finitely many defining relations. Consequently, as both $H$ and $G$ are finitely presented, $Q$ will also be finitely presented. The group $Q$ is NFQ as a quotient of the NFQ group $G$. One can check that the center of the group $Q$, obtained from [3, Thm. 1.1], is in fact trivial. However, it is easy to bypass this, by setting $A:=Q * S$ and observing that $A$ is still finitely presented, NFQ, has trivial center (as a non-trivial free product - see [24, 6.2.6]) and contains a copy of $H$.

Now, suppose that the word problem in $H$ is solvable. Note that the same is true in $S$, because the word problem is solvable in any recursively presented simple group ([19, IV.3.6]). By [3, Thm. 1.1] the group $Q$ above is hyperbolic relative to the family of subgroups, consisting of $H$ and two copies of $S$. Therefore $Q$ has solvable word problem (see [14, Thm. 3.7] or [22, Cor. 5.5]). Finally, the word problem is solvable in $A=Q * S$ by [19, IV.1.3].

Proposition 3.2. There exists a NFQ group $A_{1}$, with trivial center and finite presentation $P_{1}=\left\langle X_{1} \| R_{1}\right\rangle$, and a recursively enumerable subset of words $Z_{1}=\left\{z_{1}, z_{2}, \ldots\right\} \subset X_{1}^{*}$ such that the word problem in $A_{1}$ is solvable but the torsion problem for $Z_{1}$ in $A_{1}$ is unsolvable. 
Proof. Let $H_{0}$ be the center-by-metabelian group constructed by P. Hall in [15, p. 435]. Namely, $H_{0}$ is generated by two elements $a, b$, subject to the relations

$$
\begin{aligned}
{\left[\left[b_{i}, b_{j}\right], b_{k}\right] } & =1, \text { for } i, j, k=0, \pm 1, \pm 2, \ldots, \text { where } b_{i}:=a^{-i} b a^{i},[x, y]:=x^{-1} y^{-1} x y, \text { and } \\
c_{i, j} & =c_{i+k, j+k}, \text { for } j>i, \quad i, j, k=0, \pm 1, \pm 2, \ldots, \text { where } c_{i, j}:=\left[b_{j}, b_{i}\right] .
\end{aligned}
$$

As Hall proved in [15, p. 435], the center of $H_{0}$ is the free abelian group with free abelian basis $\left\{d_{1}, d_{2}, \ldots\right\}$, where $d_{r}:=c_{0, r}=\left[a^{-r} b a^{r}, b\right], r=1,2, \ldots$

Let $\left\langle a, b \| R_{0}\right\rangle$ be the above presentation for $H_{0}$. Clearly this presentation is recursive. Now, consider a computable (recursive) function $f: \mathbb{N} \rightarrow \mathbb{N}$ with non-recursive range $f(\mathbb{N}) \subset \mathbb{N}$. Let $H_{1}$ be the quotient of $H_{0}$ by the central subgroup $\left\langle d_{f(n)}^{n} \mid n \in \mathbb{N}\right\rangle$ where $d_{r}, r \in \mathbb{N}$, are as above. Then $H_{1}$ has the presentation

$$
\left\langle a, b \| R_{0},\left(\left[a^{-f(n)} b a^{f(n)}, b\right]\right)^{n}, n \in \mathbb{N}\right\rangle \text {. }
$$

The group $H_{1}$ will be recursively presented since $R_{0}$ is recursively enumerable and $f$ is computable.

By abusing notation, we will continue writing $a, b, b_{i}, d_{r}$ for the images of the corresponding elements of $H_{0}$ in $H_{1}$. We can solve the word problem in $H_{1}$ as follows. Given a word $w$, over the alphabet $\left\{a^{ \pm 1}, b^{ \pm 1}\right\}$, we want to determine whether $w=1$ in $H_{1}$. First we compute the sum $\varepsilon_{a}(w)$ of all exponents of $a$ in $w$. If $\varepsilon_{a}(w) \neq 0$, then $w \neq 1$ in $H_{1}$ as there is a homomorphism $\alpha: H_{1} \rightarrow\langle a\rangle$, whose kernel is generated by $b_{i}$, $i \in \mathbb{Z}$, such that $\alpha(w)=a^{\varepsilon_{a}(w)} \neq 1$. If $\varepsilon_{a}(w)=0$, then $w \in B:=\left\langle b_{i}, i \in \mathbb{Z}\right\rangle$ and we can re-write $w$ as a word $w_{1}$ in letters $b_{i}, i \in \mathbb{Z}$. If for some $i \in \mathbb{Z}, \varepsilon_{b_{i}}\left(w_{1}\right) \neq 0$, then, again, $w \neq 1$ in $H_{1}$, because its image will be non-trivial in the abelianization of $B$. Otherwise, $w$ will represent an element of the center $C:=\left\langle d_{r}, r \in \mathbb{N}\right\rangle$ of $H_{1}$, and we can re-write $w_{1}$ as a word $w_{2} \equiv d_{r_{1}}^{n_{1}} d_{r_{2}}^{n_{2}} \cdots d_{r_{l}}^{n_{l}}$, where $l \geqslant 0,1 \leqslant r_{1}<r_{2}<\cdots<r_{l}$, and $n_{j} \in \mathbb{Z} \backslash\{0\}$ for $j=1, \ldots, l$. Note that $C=\bigoplus_{r \in \mathbb{N}}\left\langle d_{r}\right\rangle$ by definition. If $l=0$ then $w=w_{2}=1$ in $H_{1}$. If $l>0$, then $w_{2}=1$ in $C$ if and only if the order of $d_{r_{j}}$ in $H_{1}$ divides $n_{j}$ for all $j=1,2, \ldots, l$. The latter can be verified as follows: for every positive divisor $m$ of $n_{j}$, we compute $f(m)$ and check if it is equal to $r_{j}$. If this happens for some divisor $m$ of $n_{j}$, then the order of $d_{r_{j}}$ in $H_{1}$ is $m$, by construction, and so $d_{r_{j}}^{n_{j}}=1$. If this is true for all $j=1, \ldots, l$, then $w=w_{2}=1$ in $H_{1}$. As each $n_{j}$ has only finitely many divisors, this can be checked in finitely many steps. Finally, if there is $j \in\{1, \ldots, l\}$ such that for every positive divisor $m$ of $n_{j}, f(m) \neq r_{j}$, then the order of $d_{r_{j}}$ in $H_{1}$ does not divide $n_{j}$, and hence $w=w_{2} \neq 1$ in $H_{1}$.

Thus $H_{1}$ is a finitely generated recursively presented group with solvable word problem. By a theorem of Clapham [10, Thm. 6], $H_{1}$ can be embedded in a finitely presented group $\mathrm{H}_{2}$ with solvable word problem. Now we can use Proposition 3.1 to embed $\mathrm{H}_{2}$ into a finitely presented NFQ group $A_{1}$, with trivial center and solvable word problem. Let $P_{1}=\left\langle X_{1} \| R_{1}\right\rangle$ be some finite presentation for $A_{1}$. Since $H_{1} \leqslant A_{1}$, the generators $a, b$ of $H_{1}$ can be represented by some words $w_{1}, w_{2}$ (respectively) in the alphabet $X_{1}^{ \pm 1}$, and hence every word in letters from $\left\{a^{ \pm 1}, b^{ \pm 1}\right\}$ can be effectively re-written in letters 
from $X_{1}^{ \pm 1}$. So, for every $r \in \mathbb{N}$ we can effectively compute a word $z_{r} \in X_{1}^{*}$ representing $d_{r}$ in $A_{1}$ and set $Z_{1}:=\left\{z_{r} \mid r \in \mathbb{N}\right\} \subset X_{1}^{*}$. By construction, $Z_{1}$ is recursively enumerable.

Suppose that the torsion problem for $Z_{1}$ in $A_{1}$ is solvable. Then for any $r \in \mathbb{N}$ we can compute the word $z_{r} \in Z_{1}$, representing $d_{r}$ in $A_{1}$, and check if $d_{r}$ has finite order in $A_{1}$. But the latter happens if and only if $r \in f(\mathbb{N})$. Thus we would be able to determine whether or not $r$ belongs to the range of $f$, contradicting to the choice of $f$. Therefore the torsion problem for $Z_{1}$ in $A_{1}$ is unsolvable and the proposition is proved.

The next statement suggests a construction which is in some sense opposite to the construction of Proposition 3.2.

Proposition 3.3. There exists a NFQ group $A_{2}$, with trivial center and finite presentation $P_{2}=\left\langle X_{2} \| R_{2}\right\rangle$, and a recursively enumerable subset of words $Z_{2}=\left\{z_{1}, z_{2}, \ldots\right\} \subset X_{2}^{*}$ such that every word from $Z_{2}$ represents an element of order at most 2 in $A_{2}$ but the word problem for $Z_{2}$ in $A_{2}$ is unsolvable.

Proof. Again, let us start with Hall's group $H_{0}$, used in the proof of Proposition 3.2 , keeping the same notation as before. Let $f: \mathbb{N} \rightarrow \mathbb{N}$ be a computable function with non-recursive range. We now let $H_{1}$ be the quotient of $H_{0}$ by the central subgroup $\left\langle d_{n}^{2}, d_{f(n)} \mid n \in \mathbb{N}\right\rangle$.

As before, $H_{1}$ will be finitely generated and recursively presented, however, the word problem in $H_{1}$ will be unsolvable (since the set $f(\mathbb{N})$ is not recursive). By the celebrated theorem of Higman [16], one can embed $H_{1}$ into a finitely presented group $H_{2}$, and applying Proposition 3.1, we can embed $H_{2}$ into a finitely presented NFQ group $A_{2}$ with trivial center.

Let $P_{2}=\left\langle X_{2} \| R_{2}\right\rangle$ be some finite presentation for $A_{2}$. Fix some words $w_{1}, w_{2} \in X_{2}^{*}$ representing the generators $a, b$ (respectively) of $H_{1}$ in $A_{2}$. Clearly there is an algorithm which takes on input a word in the alphabet $\left\{a^{ \pm 1}, b^{ \pm 1}\right\}$ and outputs a corresponding word in the alphabet $X_{2}^{ \pm 1}$ (substituting every $a$-letter by $w_{1}$ and every $b$-letter by $w_{2}$ ). For each $r \in \mathbb{N}$, let $z_{r} \in X_{2}^{*}$ be the word representing $d_{r} \in H_{1}$, obtained this way, and set $Z_{2}:=\left\{z_{r} \mid r \in \mathbb{N}\right\} \subset X_{2}^{*}$. Evidently the set of words $Z_{2}$ is recursively enumerable and every word from this set represents an element $d_{r}$, which has order at most 2 in $A_{2}$. By construction, $z_{r}=1$ in $A_{2}$ if and only if $d_{r}=1$ in $H_{1}$, which happens if and only if $r \in f(\mathbb{N})$. Since $f(\mathbb{N})$ is non-recursive, we see that the word problem for $Z_{2}$ in $A_{2}$ is unsolvable.

\section{Proofs of THE THEOREMS}

We are now ready to establish our two theorems.

Proof of Theorem 1.1. We start with the presentation $P_{1}=\left\langle X_{1} \| R_{1}\right\rangle$ of the group $A_{1}$, and the recursively enumerable set of words $Z_{1}=\left\{z_{1}, z_{2}, \ldots,\right\} \subset X_{1}^{*}$, which were constructed in Proposition 3.2. Take some infinite finitely presented simple group $B$ and fix some finite presentation $\langle Y \| S\rangle$ of it; recall that the word problem in $B$ is solvable by [19, IV.3.6]. Let $z_{0} \in X_{1}^{*}$ be the empty word. For each $r \in \mathbb{N} \cup\{0\}$, let $d_{r}$ denote 
the element of $A_{1}$ represented by the $z_{r} \in Z_{1}$; let $G:=A_{1} * B$ and let $C_{r+1}$ be the cyclic group of order $r+1$. Then the group $K_{r}:=G_{\tau_{d_{r}}} \times C_{r+1}$ has the presentation

$$
\begin{aligned}
& P_{1, r}:=\left\langle X_{1}, Y, t, u \| R_{1}, S, t^{-1} x^{-1} t z_{r}^{-1} x z_{r}, t^{-1} y^{-1} t y, u^{-1} x^{-1} u x,\right. \\
& \left.\qquad u^{-1} y^{-1} u y, u^{-1} t^{-1} u t, u^{r+1} \text {, for all } x \in X_{1} \text { and } y \in Y\right\rangle .
\end{aligned}
$$

Since the sets $X_{1}, Y, R_{1}$ and $S$ are finite, for every $r \in \mathbb{N} \cup\{0\}, P_{1, r}$ is a finite presentation of a group. Note that the presentation $P_{1,0}$ defines the group $K_{0} \cong G_{i d_{G}} \cong G \times \mathbb{Z}$.

Now, consider the class of group presentations $\mathscr{C}_{1}:=\left\{P_{1, r} \mid r \in \mathbb{N} \cup\{0\}\right\}$. We can make the following observations.

(a): the class $\mathscr{C}_{1}$ is recursively enumerable by definition.

(b): the word problem in $\mathscr{C}_{1}$ is uniformly solvable. This easily follows from the fact that the word problem in $G=A_{1} * B$ is solvable and for each $r \in \mathbb{N} \cup\{0\}$, $G \triangleleft K_{r}$ and $K_{r} / G \cong \mathbb{Z} \times C_{r+1}$.

(c): the isomorphism problem within $\mathscr{C}_{1}$ is trivially solvable. This is because for any $r \in \mathbb{N} \cup\{0\}$, the abelianization of the group $K_{r}$ is isomorphic to $\mathbb{Z} \times C_{r+1}$ (as $G$ is NFQ), hence for any $q \in \mathbb{N} \cup\{0\}, q \neq r$, the group $K_{r}$ is not isomorphic to $K_{q}$ since their abelianizations have different torsion subgroups. Thus any two distinct presentations from $\mathscr{C}_{1}$ define non-isomorphic groups.

(d): the commensurability problem within $\mathscr{C}_{1}$ is unsolvable. Indeed, since the index $\left[K_{r}: G_{\tau_{d r}}\right]=r+1$ is finite, the group $K_{r}$ is commensurable with the group $G_{\tau_{d r}}$ for each $r \in \mathbb{N} \cup\{0\}$. So, if we could decide whether $K_{r}$ is commensurable with $K_{0}$, then we would be able to decide whether $G_{\tau_{d_{r}}}$ is commensurable with $G_{i d_{G}}$, which, by Corollary 2.6, would imply that the torsion problem for $Z_{1}$ in $G=A_{1} * B$ is solvable, contradicting to the claim of Proposition 3.2 .

Thus the class of group presentations $\mathscr{C}_{1}$ satisfies all of the required properties.

Proof of Theorem 1.2. Now we start with the presentation $P_{2}=\left\langle X_{2} \| R_{2}\right\rangle$ of the group $A_{2}$, constructed in Proposition 3.3, and the recursively enumerable set of words $Z_{2}=$ $\left\{z_{1}, z_{2}, \ldots,\right\} \subset X_{2}^{*}$. Take some infinite finitely presented simple group $B$ and fix some finite presentation $\langle Y \| S\rangle$ of it; then $B$ will have solvable word problem ([19, IV.3.6]). Let $z_{0} \in X_{2}^{*}$ be the empty word. For each $r \in \mathbb{N} \cup\{0\}$, let $d_{r}$ denote the element of $A_{2}$ represented by the $z_{r} \in Z_{2}$ and let $G:=A_{2} * B$. Then the group $G_{\tau_{d_{r}}}$ has the presentation

$$
P_{2, r}:=\left\langle X_{2}, Y, t \| R_{2}, S, t^{-1} x^{-1} t z_{r}^{-1} x z_{r}, t^{-1} y^{-1} t y \text {, for all } x \in X_{2} \text { and } y \in Y\right\rangle \text {. }
$$

Since the sets $X_{2}, Y, R_{2}$ and $S$ are finite, for every $r \in \mathbb{N} \cup\{0\}, P_{2, r}$ is a finite presentation of a group. As before, the presentation $P_{2,0}$ defines the group $G_{i d_{G}} \cong G \times \mathbb{Z}$.

For the class of finite presentations $\mathscr{C}_{2}:=\left\{P_{2, r} \mid r \in \mathbb{N} \cup\{0\}\right\}$ we can observe the following.

(a): the class $\mathscr{C}_{2}$ is recursively enumerable by definition.

(b): the commensurability problem within $\mathscr{C}_{2}$ is trivially solvable, because any presentation from this class defines the group $G_{\tau_{d r}}$, for some $r \in \mathbb{N} \cup\{0\}$, which is commensurable with $G_{i d_{G}}$ by Corollary 2.6, as the element $d_{r} \in A_{2}$ has finite 
order by construction. Thus any two presentations from $\mathscr{C}_{2}$ define commensurable groups.

(c): the isomorphism problem within $\mathscr{C}_{2}$ is unsolvable. Indeed, according to Corollary 2.6. for any $r \in \mathbb{N}$, the group $G_{\tau_{d_{r}}}$, defined by $P_{2, r}$ is isomorphic to $G_{i d_{G}}$, defined by $P_{2,0}$, if and only if $z_{r}=1$ in $G$. Thus the isomorphism problem within $\mathscr{C}_{2}$ is equivalent to the word problem for $Z_{2}$ in $A_{2}$, which is unsolvable by construction.

Thus the class $\mathscr{C}_{2}$ satisfies all of the needed properties.

\section{DECISION PROBLEMS IN GEOMETRIC GROUP THEORY}

From the viewpoint of geometric group theory, besides the isomorphism and commensurability relations, there are several other equivalence relations on groups which are of natural interest:

- Two finitely generated groups $G_{1}, G_{2}$ are virtually isomorphic (sometimes also called commensurable up to finite kernels) if there exist a pair of finite index subgroups $H_{i} \leqslant G_{i}$, and some further finite normal subgroups $N_{i} \unlhd H_{i}, i=1,2$, with isomorphic quotients $H_{1} / N_{1} \cong H_{2} / N_{2}$.

- Two finitely generated groups $G_{1}, G_{2}$ are quasi-isometric if there exists a map $f: G_{1} \rightarrow G_{2}$ and a constant $K>0$ so that for all $x, y \in G_{1}$

$$
\frac{1}{K} d_{1}(x, y)-K \leqslant d_{2}(f(x), f(y)) \leqslant K \cdot d_{1}(x, y)+K
$$

and the $K$-neighborhood of $f\left(G_{1}\right)$ is all of $G_{2}$ (the $d_{i}$ are word metrics on the $G_{i}, i=1,2$ ).

- Two finitely generated groups are bi-Lipschitz equivalent if there is a bi-Lipschitz map between $\left(G_{1}, d_{1}\right)$ and $\left(G_{2}, d_{2}\right)$, where again the $d_{i}$ are word metrics (this is equivalent to the existence of a bijective quasi-isometry between them - see Whyte [32]).

We can now state the corresponding decision problems: the virtual isomorphism problem (respectively, quasi-isometry problem or bi-Lipschitz problem) asks whether there exists an algorithm which, given two finite presentations of groups, can decide whether or not they define virtually isomorphic (resp. quasi-isometric or bi-Lipschitz equivalent) groups. Several of these problems have been studied from the viewpoint of descriptive set theory by Thomas [28, 29, 30, 31]. Note that a group is bi-Lipschitz equivalent to the trivial group if and only it is trivial, and that it is virtually isomorphic, commensurable, or quasi-isometric to the trivial group if and only if it is finite. Since the problem of deciding whether a finitely presented group is finite (or trivial) is unsolvable (this follows from the famous Adian-Rabin theorem, see [2, 1] and [23]), we immediately obtain

Lemma 5.1. Within the class of all finite presentations of groups, the virtual isomorphism, quasi-isometry, bi-Lipschitz, and commensurability problems are all unsolvable. 
It would be of some interest to study the relative complexity of these various decision problems. A straightforward consequence of our construction appearing in the proof of Theorem 1.2 is the following:

Corollary 5.2. There exists a recursively enumerable class of finite presentations of groups within which the isomorphism problem is unsolvable, but the virtual isomorphism, quasi-isometry, and bi-Lipschitz problems are all (trivially) solvable.

Proof. In the notations from the proof of theorem 1.2, let $\Phi:=\left\{\tau_{d_{r}} \mid r \in \mathbb{N} \cup\{0\}\right\} \subset$ $\operatorname{Aut}(\mathrm{G})$. Then the class of finite presentations $\mathscr{C}_{2}$, constructed in the proof of Theorem 1.2, defines the class $\mathscr{K}_{G, \Phi}=\left\{G_{\tau_{d_{r}}} \mid r \in \mathbb{N} \cup\{0\}\right\}$ of finitely presented groups. As we noticed above, any two groups from this class are commensurable. And since commensurable groups are automatically quasi-isometric, all the groups in $\mathscr{K}_{G, \Phi}$ are quasi-isometric to each other, and the quasi-isometry problem within $\mathscr{C}_{2}$ is (trivially) solvable.

Moreover, none of the groups $G_{\tau_{d_{r}}}, r \in \mathbb{N} \cup\{0\}$, can contain a non-trivial finite normal subgroup, for such a subgroup would have to map to the identity under the canonical projection $G_{\tau_{d_{r}}} \rightarrow \mathbb{Z}$, and hence it would have to be a normal subgroup in the group $G=A_{2} * B$. But a non-trivial free product does not have any non-trivial finite normal subgroups. This tells us that within the class $\mathscr{K}_{G, \Phi}$, two groups are virtually isomorphic if and only if they are commensurable. Therefore the virtual isomorphism problem within $\mathscr{C}_{2}$ is also (trivially) solvable.

Finally, noting that $G=A_{2} * B$ is non-amenable, as a non-elementary free product, and embeds into every $G_{\tau_{d r}}, r \in \mathbb{N} \cup\{0\}$, we see that all the groups in the class $\mathscr{K}_{G, \Phi}$ are non-amenable. The work of Block and Weinberger [4, Thm. 3.1] implies that the groups in this class all have vanishing 0-dimensional uniformly finite homology. Whyte's thesis [32, Thm. 1.1] then implies that commensurability between any two groups from $\mathscr{K}_{G, \Phi}$ can be promoted to a bi-Lipschitz equivalence. We conclude that all the groups in $\mathscr{K}_{G, \Phi}$ are bi-Lipschitz equivalent to each other, so that the bi-Lipschitz problem within $\mathscr{C}_{2}$ is also (trivially) solvable.

More generally, we expect that these various decision problems are fundamentally unrelated to each other (with the possible exception of the bi-Lipschitz problem, in view of Whyte's thesis [32] ). To be more precise, we suspect that given any two disjoint subsets of these decision problems, one can find a recursively enumerable class of finite presentations of groups such that any problem from the first of these subsets is solvable within this class, while problems from the second subset are all unsolvable.

In another vein, these algorithmic problems are also open for various natural classes of groups. For instance, one could focus on certain classes of lattices within a fixed semi-simple Lie group $G$ of non-compact type. If the $\mathbb{R}$-rank of $G$ is $\geqslant 2$, and one restricts to uniform lattices (so that the quasi-isometry problem is trivially solvable), is the isomorphism problem or commensurability problem solvable? If one focuses on $G=S O(n, 1), n \geqslant 4$, and restrict to non-uniform lattices (so that the isomorphism problem is solvable, by Dahmani and Groves [11]), is the commensurability problem 
(equivalent to the quasi-isometry problem, by Schwartz [25]) solvable or not? Surprisingly, these questions do not seem to have been considered in the literature.

\section{Acknowledgments}

The authors thank Cornelia Druţu, Daniel Groves, and Mark Sapir for helpful conversations. The research of the first author was partially supported by the ERC grant ANALYTIC no. 259527, and by the Swiss NSF, under Sinergia grant CRSI22-130435. The second author was partially supported by the NSF, under grant DMS-0906483, and by an Alfred P. Sloan research fellowship. The work of the third author was supported by the EPSRC grant EP/H032428/1.

\section{REFERENCES}

[1] S. I. Adian. Finitely presented groups and algorithms. Dokl. Akad. Nauk SSSR, 117:9-12, 1957.

[2] S. I. Adian. The unsolvability of certain algorithmic problems in the theory of groups. Tr. Mosk. Mat. Obshch., 6:231-298, 1957.

[3] G. Arzhantseva, A. Minasyan, and D. Osin. The SQ-universality and residual properties of relatively hyperbolic groups. J. Algebra, 315:165-177, 2007.

[4] J. Block and S. Weinberger. Aperiodic tilings, positive scalar curvature and amenability of spaces. J. Amer. Math. Soc., 5:907-918, 1992.

[5] A. Borel. Commensurability classes and volumes of hyperbolic 3-manifolds. Ann. Scuola Norm. Sup. Pisa Cl. Sci. (4), 8(1):1-33, 1981.

[6] M. Burger and S. Mozes. Finitely presented simple groups and products of trees. C. R. Acad. Sci. Paris Sér. I Math., 324:747-752, 1997.

[7] M. Burger and S. Mozes. Lattices in product of trees. Inst. Hautes Études Sci. Publ. Math., 92:151194, 2000.

[8] P.-E. Caprace and B. Remy. Simplicité abstraite des groupes de Kac-Moody non affines. C. R. Math. Acad. Sci. Paris, 342:539-544, 2006.

[9] P.-E. Caprace and B. Remy. Simplicity and superrigidity of twin building lattices. Invent. Math., 176:169-221, 2009.

[10] C. R. J. Clapham. An embedding theorem for finitely generated groups. Proc. London Math. Soc. (3), 17:419-430, 1967.

[11] F. Dahmani and D. Groves. The isomorphism problem for toral relatively hyperbolic groups. Publ. Math. Inst. Hautes Études Sci., 107:211-290, 2008.

[12] M. Dehn. Über unendliche diskontinuierliche Gruppen. Math. Ann., 71:116-144, 1911.

[13] P. Deligne and G. D. Mostow. Commensurabilities among lattices in PU $(1, n)$, volume 132 of Annals of Mathematics Studies. Princeton University Press, Princeton, NJ, 1993.

[14] B. Farb. Relatively hyperbolic groups. Geom. Funct. Anal., 8:810-840, 1998.

[15] P. Hall. Finiteness conditions for soluble groups. Proc. London Math. Soc. (3), 4:419-436, 1954.

[16] G. Higman. Subgroups of finitely presented groups. Proc. Roy. Soc. Ser. A, 262:455-475, 1961.

[17] W. R. Parry J. W. Cannon, W. J. Floyd. Introductory notes on Richard Thompson's groups. Enseign. Math. (2), 42:215-256, 1996.

[18] S. Lipschutz and C. F. Miller, III. Groups with certain solvable and unsolvable decision problems. Comm. Pure Appl. Math., 24:7-15, 1971.

[19] R. C. Lyndon and P. E. Schupp. Combinatorial group theory. Springer-Verlag, Berlin, 1977. Ergebnisse der Mathematik und ihrer Grenzgebiete, Band 89.

[20] G. A. Margulis. Arithmeticity of nonuniform lattices. Funkcional. Anal. i Priložen., 7(3):88-89, 1973. 
[21] C. F. Miller, III. Decision problems for groups—-survey and reflections. In Algorithms and classifcation in combinatorial group theory (Berkeley, CA, 1989), volume 23 of Math. Sci. Res. Inst. Publ., pages 1-59. Springer, New York, 1992.

[22] D. V. Osin. Relatively hyperbolic groups: intrinsic geometry, algebraic properties, and algorithmic problems. Mem. Amer. Math. Soc., 179(843):vi+100, 2006.

[23] M. O. Rabin. Recursive unsolvability of group theoretic problems. Ann. of Math., 67:172-194, 1958.

[24] D. J. S. Robinson. A course in the theory of groups, volume 80 of Graduate Texts in Mathematics. Springer-Verlag, New York, second edition, 1996.

[25] R. E. Schwartz. The quasi-isometry classification of rank one lattices. Publ. Math. Inst. Hautes Études Sci., 82:133-168, 1995.

[26] C. L. Siegel. Symplectic geometry. Amer. J. Math., 65:1-86, 1943.

[27] J. Stillwell. Classical topology and combinatorial group theory, volume 72 of Graduate Texts in Mathematics. Springer-Verlag, New York, second edition, 1993.

[28] S. Thomas. The virtual isomorphism problem for finitely generated groups. Bull. London Math. Soc., 35:777-784, 2003.

[29] S. Thomas. On the complexity of the quasi-isometry and virtual isomorphism problems for finitely generated groups. Groups Geom. Dyn., 2:281-307, 2008.

[30] S. Thomas. The commensurability relation for finitely generated groups. J. Group Theory, 12:901909, 2009.

[31] S. Thomas and B. Velickovic. On the complexity of the isomorphism relation for finitely generated groups. J. Algebra, 217:352-373, 1999.

[32] K. Whyte. Amenability, bi-Lipschitz equivalence, and the von Neumann conjecture. Duke Math. J., 99:93-112, 1999.

University of Vienna, Faculty of Mathematics, Nordbergstraße 15, 1090 Vienna, AUSTRIA

E-mail address: goulnara.arzhantseva@univie.ac .at

The Ohio State University, Department of Mathematics, 100 Math Tower, 231 West 18Th Avenue, Columbus, OH 43210-1174, USA

E-mail address: jlafont@math.ohio-state.edu

School of Mathematics, University of Southampton, HighField CAMPus, SOUthampTON, SO17 1BJ, UNITED KINGDOM

E-mail address: aminasyan@gmail.com 